\title{
Kiwifruit fermentation drives positive gut microbial and metabolic changes irrespective of initial microbiota composition
}

Article

Accepted Version

Creative Commons: Attribution-Noncommercial-No Derivative Works 4.0

Blatchford, P., Stoklosinski, H., Walton, G., Swann, J., Gibson, G., Gearry, R. and Ansell, J. (2015) Kiwifruit fermentation drives positive gut microbial and metabolic changes irrespective of initial microbiota composition. Bioactive Carbohydrates and Dietary Fibre, 6 (1). pp. 37-45. ISSN 22126198 doi: https://doi.org/10.1016/j.bcdf.2015.07.001 Available at https://centaur.reading.ac.uk/66048/

It is advisable to refer to the publisher's version if you intend to cite from the work. See Guidance on citing.

Published version at: http://www.sciencedirect.com/science/article/pii/S221261981530005X

To link to this article DOI: http://dx.doi.org/10.1016/j.bcdf.2015.07.001

Publisher: Elsevier

All outputs in CentAUR are protected by Intellectual Property Rights law, including copyright law. Copyright and IPR is retained by the creators or other copyright holders. Terms and conditions for use of this material are defined in the End User Agreement. 


\section{www.reading.ac.uk/centaur}

\section{CentAUR}

Central Archive at the University of Reading

Reading's research outputs online 
1 Kiwifruit fermentation drives positive qut microbial and metabolic changes

2

\section{irrespective of initial microbiota composition}

Paul Blatchford, ${ }^{1,2}$ Halina Stoklosinski, ${ }^{1}$ Gemma Walton, ${ }^{2}$ Jonathan Swann, ${ }^{2}$ Glenn Gibson, ${ }^{2}$

Richard Gearry, ${ }^{3}$ Juliet Ansell, ${ }^{4}$

${ }^{1}$ The New Zealand Institute for Plant \& Food Research Limited, Private Bag 11600, Palmerston North 4442, New Zealand

${ }^{2}$ Department of Food and Nutritional Sciences, The University of Reading, Reading RG6 6AP, United Kingdom

${ }^{3}$ Department of Medicine, University of Otago, Christchurch, New Zealand

${ }^{4}$ Zespri International Limited, 400 Maunganui Road, PO Box 4043, Mt Maunganui 3149, New Zealand

\section{Abstract}

It is well established that individuals vary greatly in the composition of their core microbiota.

Despite differing ecology, we show here that metabolic capacity converges under the

4 pressure of kiwifruit substrates in a model gut system. The impact of pre-digested green and

gold kiwifruit on the human colonic microbiota and their metabolic products was assessed

using in vitro, $\mathrm{pH}$-controlled, anaerobic batch culture fermenters. Phylogenetic analyses

7 revealed that bacterial composition changed over time, irrespective of whether a substrate

8 was added or not, indicating a natural adjustment period to the gut model environment.

Adding kiwifruit substrate caused additional changes in terms of growth of specific bacterial groups, bacterial diversity and metabolite profiles. Relative abundance of Bacteroides spp. increased with both green and gold kiwifruit substrate while Bifidobacterium spp. increased only with green kiwifruit. NMR spectroscopy and GC demonstrated an increase in organic acids (primarily acetate, butyrate, propionate) and a concomitant decrease in several amino acids and oligosaccharides following addition of green and gold kiwifruit substrate. The experiments demonstrated that despite markedly different baseline profiles in individual donor inoculum, kiwifruit polysaccharides can induce substantive change in microbial ecology and metabolism which could have consequences for human health. 


\section{Introduction}

The gut microbiota is a highly diverse collection of trillions of microbes comprised of hundreds of species (Gill et al., 2006). In the densely colonised large intestine, the microbiota can reach numbers of up to $10^{12} \mathrm{cfu} / \mathrm{g}$ colon contents (Gueimonde \& Collado, 2012). Such vast numbers of symbionts can have a considerable impact on the health of the host. The gut microbiota has evolved with humans to a complex inter-dependent state, where their genome in addition to our own generates a profound ability to metabolise the diverse array of substrates in the human diet (Xu et al., 2007). Predominant phyla in the human gut are Bacteroidetes and Firmicutes, making up over $90 \%$ of all resident colonic bacteria with the two other subdominant phyla being Actinobacteria and Proteobacteria (Eckburg et al., 2005, Ley et al., 2006). There have been numerous studies conducted recently on the use of purified and processed foods or food additives to modify bacterial composition. It is clear that diet has an effect on microbiota and this in turn affects health; as many as a third of all diseases, including cardiovascular disorders such as coronary heart disease and hypertension, type 2 diabetes, functional bowel problems and cancer, are lifestyle related and their risk may be mitigated through dietary means (Tuomilehto et al., 2001, Johnson et al., 2006, Shahidi, 2009). Prebiotic supplementation is commonly used to treat gastrointestinal dysfunction. These are a class of non-digestible food ingredients such as fructo-oligosaccharides (FOS), galacto-oligosaccharides (GOS) and xylooligosaccharides (XOS) (Gibson, 2004) that confer a health benefit to the host through selectively modulating bacterial composition (Gibson et al., 2010). Prebiotic molecules are often sourced from plants, where specific oligosaccharides are isolated and concentrated in order to be used as a supplement to a regular diet. An alternative to prebiotic supplementation is the use of whole fruits and vegetables as health promoting foods, which are easier to implement into a dietary routine (Lipsky et al., 2012). In addition to the health benefits of whole foods, they make for a more marketable product. An absence of processing maintains the natural structure of nutrients which are potentially more bioavailable in whole foods (van der Sluis et al., 2002, Chandrasekara et al., 2012).

The most commonly sold kiwifruit are from the species Actinidia deliciosa (typically green fleshed e.g. 'Hayward') and A. chinensis (typically yellow fleshed e.g. 'Gold3'). Kiwifruit are rich in vitamin C, potassium, folate, and phytochemicals (Ferguson \& Ferguson, 2003). The principal carbohydrate found in kiwifruit is starch, with non-starch polysaccharides (NSP) such as pectic polysaccharides, hemicelluloses and celluloses amounting to $2-3 \%$ of total kiwifruit constituents (Dawson \& Melton, 1991, Seager \& Haslemore, 1993, Ferguson \& Ferguson, 2003, Carnachan et al., 2012). NSP are essentially resistant to digestion by enzymes encountered in the human stomach and small intestine. Therefore, they reach the 
colon largely intact where pectic polysaccharides and, to a lesser extent, hemicelluloses and celluloses are fermented by the gut microbiota (Cummings \& Englyst, 1987). Prebiotic effects, namely beneficial changes to the composition of the existing microbiota and colonic metabolites, may subsequently be observed. Several studies have examined kiwifruit fibre digestion in vitro, finding a chemically unaltered structure with only minor modifications to galacturonic acid residues and molecular weight profiles in the soluble fibre fraction (Dawson \& Melton, 1991, Carnachan et al., 2012). In a recent study, upper gastrointestinal tract digestion had little effect on either green or gold kiwifruit in an in vivo porcine model, with the dietary fibre fraction being completely undigested at the terminal ileum (Henare et al., 2012).

Changes in bacterial composition can lead to a modified metabolite profile which can have direct consequences for host health. Recent research has shown that the observed metabolic profile can be altered by changing the substrates available for fermentation. Substrates that can induce changes in metabolic profiles include: carbohydrates such as resistant starch, unabsorbed sugars, non-starch polysaccharides, gums and cellulose; and proteins from the diet and endogenous sources such as mucin (Cummings \& Englyst, 1987, Cummings \& Macfarlane, 1991, Louis et al., 2007). Some of the main end products of fermentation are short chain fatty acids (SCFA), branched chain fatty acids (BCFA) and gases like hydrogen, carbon dioxide and methane (Blaut, 2002, Rosendale et al., 2011). This study determined the effect of whole kiwifruit components that escape gastric and small intestinal digestion on the colonic microbiota and metabolites in an in vitro batch culture gut model. 


\section{Materials and Methods}

\section{Simulated Gastrointestinal Digestion (SGD)}

Two kiwifruit substrates were used in the batch culture models: Green kiwifruit (Actinidia deliciosa) 'Hayward' and Gold kiwifruit (Actinidia chinensis) 'Hort16A'. As a negative control, no exogenous substrate was added. Green and gold kiwifruit were peeled, chopped and mashed finely. The samples were subjected to the simulated gastric digestion procedure as detailed by Mills et al. with minor modifications (Mills et al., 2008). Briefly, $60 \mathrm{~g}$ of sample was weighed and added to $150 \mathrm{~mL}$ of autoclaved distilled water in a stomacher bag where it was homogenised (Stomacher 400, Seward, West Sussex, UK) for 5 min at normal speed (460 paddle beats / min). After addition of $0.001 \mathrm{~mol} / \mathrm{L}$ salivary $\alpha$-amylase the solution was incubated for $30 \mathrm{~min}$ on a shaker at $37^{\circ} \mathrm{C}$. The $\mathrm{pH}$ was adjusted to 2.0 using $6 \mathrm{M} \mathrm{HCl}$. Pepsin solution was added to the mixture which was incubated at $37^{\circ} \mathrm{C}$ gently shaking for 2 h. The $\mathrm{pH}$ was adjusted to 7.0 following addition of a pancreatin/bile mixture (P8096/B8631 Sigma, Poole, Dorset, UK) and the solution was incubated at $37^{\circ} \mathrm{C}$ for $3 \mathrm{~h}$. Samples were then transferred to a 500 Da dialysis membrane (Spectra/Por, Spectrum Laboratories Inc., $\mathrm{UK}$ ) to remove most di- and mono-saccharides. This was dialysed for $15 \mathrm{~h}$ against a $10 \mathrm{mM}$ $\mathrm{NaCl}$ solution at $4{ }^{\circ} \mathrm{C}$. The dialysis fluid was replenished and the samples dialysed for a further $2 \mathrm{~h}$. Samples were then frozen at $-80^{\circ} \mathrm{C}$ and freeze-dried.

\section{pH controlled anaerobic faecal batch cultures}

Batch culture systems allow the study of microbial fermentation in a simulated colonic environment. The apparatus was set up the day before the experiment and sterilised by autoclaving. The basal culture medium used for the batch cultures contained (per L): $2 \mathrm{~g}$ peptone, $2 \mathrm{~g}$ yeast extract, $0.1 \mathrm{~g} \mathrm{NaCl}, 0.04 \mathrm{~g} \mathrm{~K}_{2} \mathrm{HPO}_{4}, 0.04 \mathrm{~g} \mathrm{KH}_{2} \mathrm{PO}_{4}, 0.01 \mathrm{~g} \mathrm{MgSO}_{4} \cdot 7 \mathrm{H}_{2} \mathrm{O}$, $0.01 \mathrm{~g} \mathrm{CaCl}_{2} \cdot 6 \mathrm{H}_{2} \mathrm{O}, 2 \mathrm{~g} \mathrm{NaHCO}_{3}, 2 \mathrm{~mL}$ Tween 80, $0.05 \mathrm{~g}$ haemin (dissolved in a few drops of $\mathrm{NaOH}), 10 \mu \mathrm{l}$ vitamin $\mathrm{K}, 0.5 \mathrm{~g}$ cysteine $\mathrm{HCl}, 4 \mathrm{~mL}$ resazurin solution $(0.025 \mathrm{~g} / 100 \mathrm{~mL})$ and $0.5 \mathrm{~g}$ No.3 bile salts. The solution was made up to $1 \mathrm{~L}$ with distilled water and sterilised by autoclaving. All chemicals were obtained from Sigma (Poole, Dorset, UK). One hundred and thirty-five millilitres of freshly autoclaved medium was aseptically poured into $280 \mathrm{~mL}$ capacity water-jacketed batch culture vessels. The medium was continually mixed using a magnetic stirrer and maintained at $37^{\circ} \mathrm{C}$ with a circulating waterbath. Oxygen free $\mathrm{N}_{2}$ gas was bubbled through the media overnight to establish an anoxic environment. Excess gas was vented outside through a $0.22 \mu \mathrm{m}$ filter.

On the morning of the experiment, calibrated $\mathrm{pH}$ electrodes were inserted into each vessel. A freshly voided stool sample was obtained from a healthy volunteer who had not taken any 
supplemental probiotics, prebiotics or antibiotics for 6 months prior. The stool was diluted 1:10 in sterile PBS, stomached for $2 \mathrm{~min}$ and $15 \mathrm{~mL}$ was added to the vessels, yielding a total volume in each vessel of $150 \mathrm{~mL}$. Then $1.5 \mathrm{~g}(1 \% \mathrm{w} / \mathrm{v})$ of each kiwifruit substrate was added to the vessels (excluding the negative control). Approximately $5.5 \mathrm{~mL}$ of sample was taken from each vessel immediately upon addition of substrate representing the $0 \mathrm{~h}$ time point. Samples were then taken at 5, 10, 24 and $48 \mathrm{~h}$ time points. Each sample was placed on ice, dispensed into aliquots and stored appropriately. The batch culture systems were monitored throughout the $48 \mathrm{~h}$ run, with any adjustments of stirrer speed, $\mathrm{N}_{2}$ flow rates or temperature carried out as required. This initial batch culture experiment was repeated twice with different faecal donors giving a total of three biological replicates.

\section{Nuclear Magnetic Resonance (NMR) Spectroscopy}

One millilitre of fermenta was taken and centrifuged at 16,200 $\mathrm{g}$ for $10 \mathrm{~min}$; then the supernatant was decanted and frozen at $-80^{\circ} \mathrm{C}$ until analysis. Samples were then defrosted, vortexed and $400 \mu \mathrm{L}$ transferred into a sterile eppendorf. Two hundred microlitres of phosphate buffer (containing $1 \mathrm{mM}$ of the internal standard TSP (3-(trimethylsilyl)-[2,2,3,3d4]-propionic acid sodium salt), the bacteriostatic sodium azide in $100 \% \mathrm{D}_{2} \mathrm{O}$ ) was added to the samples which were then vortexed and centrifuged at $10,000 \mathrm{~g}$ for $10 \mathrm{~min}$. The supernatant $(550 \mu \mathrm{L})$ was then transferred to a $5 \mathrm{~mm}$ glass NMR tube. All samples (and a batch culture medium only control) were run on a Bruker Avance III $700 \mathrm{MHz}$ NMR spectrometer. Initial spectral processing was conducted using Bruker's Topspin software. Spectra were baseline corrected to remove systemic offsets, phased to yield accurate peak integration and peak shape and the TSP (internal chemical shift standard) adjusted to 0 ppm. Further data processing was carried out using MATLAB 7.8.0 R2013b. The spectral regions containing the resonances from residual water and polyethylene glycol were removed to minimise the effects of baseline distortions. Principal Component Analysis (PCA) was performed on the metabolic profiles in Matlab using scripts provided by Korrigan Sciences Ltd., United Kingdom. This unsupervised approach was used to determine inherent changes between batch culture samples of different substrates or at different time-points.

\section{Gas Chromatography}

Concentrations of acetate, butyrate, formate, heptanoate, hexanoate, isobutyrate, isovalerate, lactate, propionate, succinate and valerate were quantified by gas chromatography equipped with a flame ionization detector (GC-FID) following a modified method of Richardson et al. (Richardson et al., 1989). In brief, $1.5 \mathrm{~mL}$ of sample was centrifuged at $16,200 \mathrm{~g}$ for $10 \mathrm{~min}$. The supernatant was diluted in $0.01 \mathrm{M}$ phosphate buffered saline with 2-ethylbutyric acid ( $5 \mathrm{mM})$ as an internal standard. The sample was then 
centrifuged at $3000 \mathrm{~g}$ for $5 \mathrm{~min}\left(4^{\circ} \mathrm{C}\right)$. The clarified supernatant was acidified with concentrated hydrochloric acid and diethyl ether added, and following vortexing, was centrifuged at $10,000 \mathrm{~g}$ for $5 \mathrm{~min}\left(4^{\circ} \mathrm{C}\right)$. The upper diethyl ether phase was collected and derivatised with $\mathrm{N}$-tert-butyldimethylsilyl-N-methyltrifluoroacetamide with $1 \%$ tertbutyldimethylchlorosilane (MTBSTFA + TBDMSCI, 99:1; Sigma-Aldrich) by heating to $80^{\circ} \mathrm{C}$ for $20 \mathrm{~min}$. To allow complete derivatisation, the samples were left for $48 \mathrm{~h}$ at room temperature before analysis. Standards containing 2-ethylbutyric acid (5 mM) as an internal standard were prepared alongside the samples. Analysis was performed on a Shimadzu gas chromatograph system (GC-17A, Kyoto, Japan) equipped with a flame ionization detector and fitted with a HP-1 column $(10 \mathrm{~m} \times 0.53 \mathrm{~mm}$ ID $\times 2.65 \mu \mathrm{m})($ Agilent Technologies, Santa Clara, CA, USA). The carrier gas was helium with a total flow rate of $37 \mathrm{~mL} / \mathrm{min}$ and pressure of $7 \mathrm{kPa}$. The temperature profile began at $70^{\circ} \mathrm{C}$, increasing to $80^{\circ} \mathrm{C}$ at $10^{\circ} \mathrm{C} / \mathrm{min}$, with a final increase to $255^{\circ} \mathrm{C}$ at $20^{\circ} \mathrm{C} / \mathrm{min}$, holding for $5 \mathrm{~min}$. The pressure program was set to $7 \mathrm{kPa}$, increasing to $15 \mathrm{kPa}$ at $0.8 \mathrm{kPa} / \mathrm{min}$, holding for $4 \mathrm{~min}$. Injector and detector temperatures were set at $260{ }^{\circ} \mathrm{C}$. Samples were injected $(1 \mu \mathrm{L})$ with a splitless injection. The instrument was controlled and chromatograms acquired using GC Solution Chromatography Data System software, Version 2.3 (Shimadzu). The acquired GC data were used to plot standard curves and calculate compound response factors in relation to the internal standard, enabling a sample result of $\mu \mathrm{mol}$ SCFA $/ \mathrm{mL}$ fermentation supernatant to be calculated.

\section{DNA Extraction, 16S rRNA gene sequencing and bioinformatics}

A $1.5 \mathrm{~mL}$ sample aliquot was centrifuged at $16,200 \mathrm{~g}$ for $10 \mathrm{~min}$. The pellet was resuspended in $500 \mu \mathrm{L}$ sterile $50 \%$ glycerol in PBS $(\mathrm{v} / \mathrm{v})$ and frozen at $-20^{\circ} \mathrm{C}$ until analysis. This sample was centrifuged at $16,200 \mathrm{~g}$ for $5 \mathrm{~min}$ and the pellet washed in $1 \mathrm{~mL}$ of PBS. The sample was centrifuged again at $16,200 \mathrm{~g}$ for $5 \mathrm{~min}$ and the pellet resuspended in $500 \mu \mathrm{L}$ of TES buffer pH 8.0 (Trizma HCl $0.254 \mathrm{~g}$, Trizam base $0.048 \mathrm{~g}$, NaCl $0.116 \mathrm{~g}$, EDTA $0.068 \mathrm{~g}$, sterile distilled water $400 \mathrm{~mL}$ ). The samples were placed on ice and $8 \mu \mathrm{L}$ lysozyme (10 $\mathrm{mg} / \mathrm{mL})$ and $2 \mu \mathrm{L}$ mutanolysin $(1 \mathrm{mg} / \mathrm{mL})$ were added. The cell suspension was vortexed and incubated at $37^{\circ} \mathrm{C}$ for $30 \mathrm{~min}$. The samples were removed and placed on ice where 10 $\mu \mathrm{L}$ proteinase $\mathrm{K}(20 \mathrm{mg} / \mathrm{mL})$ and $10 \mu \mathrm{L}$ Rase $(10 \mathrm{mg} / \mathrm{mL})$ were added. The cell suspension was vortexed and incubated at $65^{\circ} \mathrm{C}$ for $1 \mathrm{~h}$. Then $100 \mu \mathrm{L}$ of $10 \%$ SDS was added and the tubes were gently mixed by inversion. The samples were then incubated for a further $15 \mathrm{~min}$ at $65^{\circ} \mathrm{C}$. The samples were cooled on ice for $30 \mathrm{~min}$ and then $620 \mu \mathrm{L}$ phenol/chloroform/water mix was added to the samples. The tubes were gently mixed by inversion for $2 \mathrm{~min}$ and then centrifuged at 4,100 $\mathrm{g}$ for $10 \mathrm{~min}$. The upper (aqueous) layer 
was transferred to a clean eppendorf tube and $1 \mathrm{~mL}$ of ice-cold ethanol was added. The samples were left on ice for 30 min or stored overnight at $-20^{\circ} \mathrm{C}$. The samples were centrifuged for $5 \mathrm{~min}$ at $16,200 \mathrm{~g}$, the supernatant was carefully removed and the pellet allowed to air dry for 2-3 h or overnight. The pellet was resuspended in $50 \mu \mathrm{L}$ sterile $\mathrm{H}_{2} \mathrm{O}$, mixed well and DNA purity and concentration was assessed by running $2 \mu \mathrm{L}$ on the NanoDrop ND-100 spectrophotometer.

DNA was used as a template to amplify variable regions V2-V3 of the 16S rRNA gene (position 336-535 in the Escherichia coli rRNA gene) using primers HDA-1 (cgtatcgectccctcgcgccatcagACTCCTACGGGAGGCAGCAGT) and HDA-2 (ctatgcgccttgccagcccgctcagNNNNNNNNNNGTATTACCGCGGCTGCTGGCAC) (Rosendale et al., 2012) where the sequences of the forward and reverse primers are shown in lower case, the four base library "key" sequence is underlined, the letter $N$ denotes the 10 base barcode sequence and the remaining capital letters the template-specific HDA primers (Tannock et al., 2000). Twenty-five microlitres of HotStarTaq master mix (Qiagen, Melbourne, Australia) was mixed with $1 \mu \mathrm{L}$ template DNA and $100 \mathrm{nM}$ of each primer (total reaction volume $50 \mu \mathrm{L}$ ). PCR conditions were as follows: Initial denaturation $95^{\circ} \mathrm{C}$ for $15 \mathrm{~min}$ then thirty cycles of $1 \min 95^{\circ} \mathrm{C}$ denaturation, $45 \sec 65^{\circ} \mathrm{C}$ annealing, $1 \min 72^{\circ} \mathrm{C}$ extension. The PCR products were gel purified using the QIAquick PCR purification kit, (QIAGEN, Melbourne, Australia), quantified using the Qubit 2.0 fluorometer (Life Technologies), pooled in equimolar quantities, and submitted for sequencing on the Roche 454 GS FLX Titanium platform (Macrogen Inc., Korea).

QIIME software V1.8.0 was used to analyse the 454 sequencing data (Caporaso et al., 2010). Reads were clustered into operational taxonomic units (OTUs) based on a $97 \%$ identity threshold value. Alignment of the sequences was carried out using PyNAST (Caporaso et al., 2010) with reference to the Greengenes core reference database (version 13_8) (DeSantis et al., 2006). Taxonomic assignment was made using the RDP Naive Bayesian classifier (Wang et al., 2007). Chimeric sequences were removed from the reads using the ChimeraSlayer algorithm (Haas et al., 2011, Claesson et al., 2012). Alpha rarefaction was calculated using the Phylogenetic Diversity (PD) whole tree, Chao1, Observed Species and Shannon diversity metrics. Beta diversity was determined using UniFrac distances as input and EMPeror to visualise relationships in three dimensions (Vazquez-Baeza et al., 2013). 


\section{Statistical Analysis}

240 ANOVA for determining differences in OTU frequencies between sample groups was

241 conducted in QIIME using the group_significance.py script. The non-parametric two-sample

242 t-test (Monte Carlo permutation) from QIIME was used for determining alpha diversity

243 significance. All other statistical calculations were conducted in R Studio using the vegan

244 and made4 packages (Culhane et al., 2005, RStudio, 2012, Jari Oksanen, 2013). The

245 Wilcoxon/Mann-Whitney test was conducted to assess differences between taxa at the

246 phylum, family and genus level. A $P$-value of less than 0.05 was deemed significant and the

247 False Discovery Rate (FDR) method was used for correcting for multiple comparisons. 
Results

The V2-V3 hypervariable region of the 16S rRNA gene sequencing results yielded 253,852 reads that passed the quality filters at an average of $5903 \pm 1703$ reads per sample. Reads were clustered into non-chimeric OTUs based on a $97 \%$ identity threshold value. Inspection of the relative abundance data at phylum and genus levels (Figure 1) indicated that the substrates had distinct effects on the microbial ecology within the in vitro fermentations. Bacterial profiles at time 0 for both substrates and control were very similar (Figure S1) and form a baseline from which changes over time caused by fermentable substrate can be measured. Several bacterial groups increased in abundance in all three vessels over the course of the experiment including Enterobacteriaceae, Sutterella spp., Veillonella spp., Collinsella spp., and Citrobacter spp.. Genera which decreased in abundance, irrespective of vessel, over time were Faecalibacterium spp., Blautia spp., Prevotella spp. and Lachnospira spp.. Bifidobacterium spp. increased when the vessel was supplemented with green kiwifruit digesta (Figure 1B). This enrichment was observable despite the $P$-value falling short of significance after FDR correction ( $P-0.2$ for $0 \mathrm{~h}$ vs $24 \mathrm{~h}$ and $P-0.4$ for $0 \mathrm{~h}$ vs $48 \mathrm{~h}$ ). This is most likely due to the variation in response to the treatment between donors, which is quite common and is also demonstrated in the beta diversity biplots (Figure 2). For example, donor 1 had a significantly higher occurrence of bifidobacterial OTU denovo1575 than donors 2 and $3(P-<0.001)$. Bacteroides spp. multiplied in the green and gold kiwifruit vessels after 5 to $10 \mathrm{~h}$ and then dropped back by $48 \mathrm{~h}$, whereas Bacteroides spp. in the negative vessel remained relatively constant (Figure 1C). Although barely detectable in the kiwifruit vessels, Oscillospira spp. became prominent in the negative vessel, reaching almost $5 \%$ of total abundance. At species-level, it was found that Faecalibacterium prausnitzii accounted for the majority of the Faecalibacterium species which dropped from about $13 \%$ of total abundance at time 0 to less than $2 \%$ by $24 \mathrm{~h}$ in all 3 vessels. Conversely, Collinsella aerofaciens increased in all three vessels and was the predominant Collinsella species.

Alpha diversity analysis was calculated by time, fermentation and substrate using four rarefaction metrics (PD whole tree, Chao1, Observed species and Shannon diversity) at 2800 reads rarefaction depth. No significant associations were evident when plotted by time or fermentation, but a clear trend towards higher diversity in the negative control and lower diversity in the gold kiwifruit vessel was observed (see Figure S2). For the PD whole tree metric, gold kiwifruit had significantly lower species richness than the negative control $(P-$ 0.003 ) at an average of $15.84 \pm 1.59$ (gold kiwifruit) vs $17.56 \pm 0.93$ (negative) as assessed by the nonparametric two-sample t-test (Monte Carlo permutation). 
When the diversity of the bacterial populations between vessels was examined (beta diversity), it was evident that separation was greatest when plotted by fermentations on a principal coordinates analysis (PCoA) plot (Figure 2); this demonstrated how samples were grouped by donor. In the PCoA plots an ordination method was used to plot variance against orthogonal axes. When the PCoA was overlaid with the ten family level bacterial groups most responsible for causing variance using the biplot function, it showed that Coriobacteriaceae, Clostridiales, Ruminococcaceae and Lachnospiraceae were the core taxa changing in the vessels. When plotted by time (Figure 2), it was clear that as time progressed the points moved from the upper right space of each of the three fermentations towards the bottom left area of the plot. This direction of migration indicates an increase in the Bacteroidaceae, Alcaligenaceae, Veillonellaceae and Enterobacteriaceae. When comparing samples as a function of substrate, there was a tendency for the negative control to cluster close to the time 0 point (data not shown).

\section{NMR Spectroscopy}

Principal components analysis (PCA) was applied to visualise the metabolic evolution of the fermentation supernatant over time (Figure 3). Principal components 1 (PC1) and 2 (PC2) collectively accounted for $77 \%$ of the variablility of the data. A time-dependent shift was observed in the scores from PC1 (horizontal movement in the scores plot; Figure 3A) and the loadings for PC1 (Figure 3B) indicate that oligosaccharides of various chain lengths are the main variables contributing to this shift. At the $0 \mathrm{~h}$ timepoint, the metabolic profiles from vessels containing kiwifruit contained a greater amount of oligosaccharides than the negative control. At this time point substrate has been added, indicating that kiwifruit contain a higher abundance of oligosaccharides than the negative control. Over time the metabolic profiles of the green and gold kiwifruit fermentations followed similar trajectories (negative PC1 and positive PC2 movement) with increased production of acetate (in agreement with the organic acid data shown in Figure 4). Propionate were also produced as the fermentation progressed, whereas lactate, leucine, alanine, succinate, and histidine decreased over time. In contrast, a minimal time-dependent shift was observed in the metabolic profiles of the negative control fermentation.

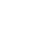


317 Concentrations of acetate and propionate increased throughout the batch culture runs in the

318 kiwifruit vessels and to a lesser extent in the negative control vessel (Figure 4). Butyrate also

319 increased over the course of the experiment, but at a similar rate for all substrates including

320 the negative control. Formic acid, succinic acid and lactic acid were liberated at the 5 and 10

321 h time points, and concentrations decreased thereafter (Figure 4). Interestingly, the vessel

322 that was not supplemented with any additional carbohydrate (negative control) had a higher

323 production of valerate and the BCFAs isobutyrate and isovalerate (Figure 4). 


\section{Discussion}

This study demonstrated that non-digestible components from green and gold kiwifruit can be utilised as fermentable substrates, effecting change to both bacterial composition and metabolism. The microbial profiles at time 0 for all three vessels were relatively similar but after 5-10 h Enterobacteriaceae and Collinsella spp. begin to markedly increase while Faecalibacterium spp. and Blautia spp. decreased, irrespective of the presence or absence of substrate. As these changes were also observed in the negative control, it is more likely a function of the gut model system, rather than a substrate response. There were unfermented substrates present within the faecal inoculum and the medium to a small extent, which may have contributed to the initial changes in fermentation seen in the model. We note that early proliferation of Enterobacteriaceae was also observed by a group running the simulator of the intestinal microbial ecosystem (SHIME) models to measure the impact of polyphenols on the human gut microbiota (Kemperman et al., 2013). Despite these background changes, the green and gold kiwifruit substrates exerted an additional powerful influence. Bifidobacterium spp. increased in abundance after $24 \mathrm{~h}$ of fermentation following exposure to green kiwifruit polysaccharides. Bacteroides spp. increased in relative abundance in response to the kiwifruit substrates prior to reverting to control levels. The genus Bacteroides include species that have a diverse array of substrate utilisation machinery encoded on polysaccharide utilisation loci (PUL) (Martens et al., 2008). Potentially, the easily fermentable substrate was metabolised in the first few hours, after which the Bacteroides were able to engage PULs to utilise the more recalcitrant substrates, thereby increasing their relative abundance. These increases in Bifidobacterium spp. and Bacteroides spp. are consistent with previously published green and gold kiwifruit fermentation results (Parkar et al., 2012).

Interestingly, the alpha diversity was lower in the vessel with the gold kiwifruit than the vessel containing the green substrate or the negative vessel. However, this occurrence is most likely due to the large bloom of Enterobacteriaceae in the gold kiwifruit vessel which were unable to be taxonomically classified below the family level. The beta diversity analyses showed a clear partitioning when calculated by donor and a distinct pattern of movement as the fermentation progressed. It is clear that different faecal donors had a considerable impact on the starting point and successive fermentation direction. This effect has been observed in experiments conducted with kiwifruit polysaccharides previously (Rosendale et al., 2012). It is not unexpected that the diversity and composition between donors varies considerably as has been shown in many studies of gut microbial ecology (Eckburg et al., 2005, Ley et al., 2006, Arumugam et al., 2011, Rosendale et al., 2012). 
378 However, despite the variability between donors, there were still consistent trends in the modulation of the microbiota.

Acetate was the most prevalent SCFA produced in all three fermentations. Acetate is absorbed into the bloodstream and used by peripheral tissue and muscle (Wong et al., 2006) and is a major metabolite of bifidobacteria (Wolfe, 2005). Propionate, butyrate and ethanol were also seen to increase over time. Organic acids are quantitatively the most abundant end-products of microbial fermentation in the human colon and their production lowers $\mathrm{pH}$ and directly inhibits the growth of pathogens (Cummings \& Englyst, 1987, Blaut, 2002). The accumulation of propionate and butyrate over time was expected as these cannot be used by cross-feeding bacteria (Louis et al., 2007). Although acetate can be metabolised, it tends to accrue over time, the generation of this oxidised molecule being an energy favourable reaction (Macfarlane \& Macfarlane, 2003). Given the comparable rates of butyrate production in the kiwifruit vessels compared with the negative control, it can be concluded that kiwifruit polysaccharides are not particularly butyrogenic. Green and gold kiwifruit substrates stimulated an increase in succinate and lactate production in the first several hours post inoculation before dropping back by the $24 \mathrm{~h}$ and $48 \mathrm{~h}$ time points. These intermediates can serve as substrate for other bacteria and may have been further converted to acetate or propionate (Louis et al., 2007). Like succinate and lactate, formate also behaved in this intermediate manner. This could be explained by the onward conversion of formate to methane which can be performed by methanogens such as Methanobrevibacter smithii or to acetate by acetogens such as Blautia hydrogenotrophica (Flint et al., 2012).

Protein only accounts for about $1 \%$ of the edible portion of green kiwifruit and a considerable proportion of this would be unable to make it to the colon without being digested or absorbed in the small intestine (Ferguson \& Ferguson, 2003). However, small amounts of protein are included in the medium, plus low levels of protein may have been introduced along with the faecal inoculum and turnover of microbes could also add to amino acids available as substrates. The BCFAs, isovalerate and isobutyrate are products of the fermentation of branched chain amino acids such as leucine, isoleucine and valine which are potentially available in the fermentation medium (Macfarlane \& Macfarlane, 2003). The negative control produced more BCFA than the kiwifruit vessels: this could indicate that microbiota were scavenging any available substrate as they would in the carbohydrate-deficient distal colon. Given that protein fermentation is an undesirable phenomenon which can lead to detrimental health effects, the lower level of BCFA production associated with kiwifruit fermentations is a positive outcome (Mortensen et al., 1992, Nyangale et al., 2012). It is not clear exactly which components of kiwifruit NSP were responsible for the changes observed in this study. 
414 Certain differences exist between green and gold kiwifruit constituents; for example, gold

415 kiwifruit is higher in hemicellulosic polysaccharides and lower in pectic polysachharides than 416 green kiwifruit (Sauvageau et al., 2010). The greater amount of hemicellulose in gold kiwifruit 417 may be responsible for stimulating the Enterobacteriaceae, resulting in a lower abundance of 418 bifidobacteria. More complex investigations are warranted that take into account the different 419 microbial niches encountered in the large bowel and the fermentation of other sources of 420 carbohydrates such as host mucin (Macfarlane et al., 2005)..

421 In summary, we have shown that kiwifruit non-digestible polysaccharides have a number of 422 effects on gut microbial ecology. The starting population of bacteria in the inoculum varies 423 greatly between donors and has a considerable impact on the subsequent fermentation 424 trajectory. However, notwithstanding the initial composition, consistent changes in microbial 425 composition and metabolite production were facilitated by kiwifruit NSP fermentation. These 426 changes included increasing Bifidobacterium spp. and Bacteroides spp. and beneficial 427 metabolites such as organic acids, which may have positive consequences for human 428 health.

\section{Acknowledgements}

430 Simone Rampelli for advice and help with bioinformatics, Adele Costabile for help with the 431 batch culture gut models and Doug Rosendale for advice on interpretation of results.

432 Authors have no conflict of interest to declare. 
444 Arumugam M, Raes J, Pelletier E, et al. (2011) Enterotypes of the human gut microbiome. Nature 445 473: 174-180.

446 Blaut M (2002) Relationship of prebiotics and food to intestinal microflora. European Journal of 447 Nutrition 41: 11-16.

448 Caporaso JG, Bittinger K, Bushman FD, DeSantis TZ, Andersen GL \& Knight R (2010) PyNAST: a flexible tool for aligning sequences to a template alignment. Bioinformatics 26: 266-267.

Caporaso JG, Kuczynski J, Stombaugh J, Bittinger K, Bushman FD, Costello EK, Fierer N, Pena AG, Goodrich JK \& Gordon JI (2010) QIIME allows analysis of high-throughput community sequencing data. Nature methods 7: 335-336.

Carnachan SM, Bootten TJ, Mishra S, Monro JA \& Sims IM (2012) Effects of simulated digestion in vitro on cell wall polysaccharides from kiwifruit (Actinidia spp.). Food Chem 133: 132-139.

Chandrasekara A, Naczk M \& Shahidi F (2012) Effect of processing on the antioxidant activity of millet grains. Food Chem 133: 1-9.

Claesson MJ, Jeffery IB, Conde S, et al. (2012) Gut microbiota composition correlates with diet and health in the elderly. Nature 488: 178-184.

459 Culhane AC, Thioulouse J, Perriere G \& Higgins DG (2005) MADE4: an R package for multivariate 460 analysis of gene expression data. Bioinformatics 21: 2789-2790.

461 Cummings JH \& Englyst HN (1987) Fermentation in the human large-intestine and the available substrates. American Journal of Clinical Nutrition 45: 1243-1255.

Cummings JH \& Macfarlane GT (1991) The control and consequences of bacterial fermentation in the human colon. Journal of Applied Bacteriology 70: 443-459.

Dawson DM \& Melton LD (1991) 2 pectic polysaccharides from kiwifruit cell-walls. Carbohydr Polym 15: 1-11.

DeSantis TZ, Hugenholtz P, Larsen N, Rojas M, Brodie EL, Keller K, Huber T, Dalevi D, Hu P \& Andersen GL (2006) Greengenes, a chimera-checked 16S rRNA gene database and workbench compatible with ARB. Appl Environ Microbiol 72: 5069-5072.

Eckburg PB, Bik EM, Bernstein CN, Purdom E, Dethlefsen L, Sargent M, Gill SR, Nelson KE \& Relman DA (2005) Diversity of the human intestinal microbial flora. Science 308: 1635-1638.

Ferguson AR \& Ferguson LR (2003) Are kiwifruit really good for you? Proceedings of the Fifth International Symposium on Kiwifruit,(Huang HW, ed.) p.^^p. 131-138.

Flint HJ, Scott KP, Louis P \& Duncan SH (2012) The role of the gut microbiota in nutrition and health. Nature Reviews Gastroenterology \& Hepatology 9: 577-589.

Gibson GR (2004) Fibre and effects on probiotics (the prebiotic concept). Clinical Nutrition Supplements 1: 25-31.

Gibson GR, Scott KP, Rastall RA, et al. (2010) Dietary prebiotics: current status and new definition. Food Science and Technology Bulletin: Functional Foods 7: 1-19.

Gill SR, Pop M, DeBoy RT, Eckburg PB, Turnbaugh PJ, Samuel BS, Gordon JI, Relman DA, FraserLiggett CM \& Nelson KE (2006) Metagenomic analysis of the human distal gut microbiome. Science 312: $1355-1359$.

Gueimonde M \& Collado MC (2012) Metagenomics and probiotics. Clin Microbiol Infect 18: 32-34. Haas BJ, Gevers D, Earl AM, et al. (2011) Chimeric 16S rRNA sequence formation and detection in Sanger and 454-pyrosequenced PCR amplicons. Genome research 21: 494-504.

Henare SJ, Rutherford SM, Drummond LN, Borges V, Boland MJ \& Moughan PJ (2012) Digestible nutrients and available (ATP) energy contents of two varieties of kiwifruit (Actinidia deliciosa and Actinidia chinensis). Food Chem 130: 67-72. Jari Oksanen FGB, Roeland Kindt, Pierre Legendre, Peter R. Minchin, R. B. O'Hara, Gavin L. Simpson, Peter Solymos, M. Henry H. Stevens and Helene Wagner (2013) vegan: Community Ecology Package. $\mathrm{R}$ package version 2.0-10. http://CRAN.R-project.org/package=vegan. 
Johnson SK, Chua V, Hall RS \& Baxter AL (2006) Lupin kernel fibre foods improve bowel function and beneficially modify some putative faecal risk factors for colon cancer in men. British Journal of Nutrition 95: 372-378.

Kemperman RA, Gross G, Mondot S, Possemiers S, Marzorati M, Van de Wiele T, Dore J \& Vaughan EE (2013) Impact of polyphenols from black tea and red wine/grape juice on a gut model microbiome. Food Research International 53: 659-669.

Ley RE, Peterson DA \& Gordon JI (2006) Ecological and evolutionary forces shaping microbial diversity in the human intestine. Cell 124: 837-848.

Ley RE, Turnbaugh PJ, Klein S \& Gordon JI (2006) Microbial ecology - Human gut microbes associated with obesity. Nature 444: 1022-1023.

Lipsky LM, Cheon K, Nansel TR \& Albert PS (2012) Candidate measures of whole plant food intake are related to biomarkers of nutrition and health in the US population (National Health and Nutrition Examination Survey 1999-2002). Nutrition research 32: 251-259.

Louis P, Scott KP, Duncan SH \& Flint HJ (2007) Understanding the effects of diet on bacterial metabolism in the large intestine. Journal of Applied Microbiology 102: 1197-1208.

Macfarlane S \& Macfarlane GT (2003) Regulation of short-chain fatty acid production. Proceedings Nutrition Society 62: 67-72.

Macfarlane S, Woodmansey EJ \& Macfarlane GT (2005) Colonization of mucin by human intestinal bacteria and establishment of biofilm communities in a two-stage continuous culture system. Appl Environ Microbiol 71: 7483-7492.

Martens EC, Chiang HC \& Gordon JI (2008) Mucosal glycan foraging enhances fitness and transmission of a saccharolytic human gut bacterial symbiont. Cell host \& microbe 4: 447-457. Mills DJS, Tuohy KM, Booth J, Buck M, Crabbe MJC, Gibson GR \& Ames JM (2008) Dietary glycated protein modulates the colonic microbiota towards a more detrimental composition in ulcerative colitis patients and non-ulcerative colitis subjects. Journal of Applied Microbiology 105: 706-714. Mortensen PB, Clausen MR, Bonnen H, Hove H \& Holtug K (1992) Colonic fermentation of ispaghula, wheat bran, glucose, and albumin to short-chain fatty acids and ammonia evaluated in vitro in 50 subjects. Journal Parenteral and Enteral Nutrition 16: 433-439.

Nyangale EP, Mottram DS \& Gibson GR (2012) Gut Microbial Activity, Implications for Health and Disease: The Potential Role of Metabolite Analysis. Journal of Proteome Research 11: 5573-5585. Parkar SG, Rosendale D, Paturi G, Herath TD, Stoklosinski H, Phipps JE, Hedderley D \& Ansell J (2012) In vitro utilization of gold and green kiwifruit oligosaccharides by human gut microbial populations. Plant foods for human nutrition 67: 200-207.

Richardson AJ, Calder AG, Stewart CS \& Smith A (1989) Simultaneous determination of volatile and non-volatile acidic fermentation products of anaerobes by capillary gas-chromatography. Lett Appl Microbiol 9: 5-8.

Rosendale D, Cookson A, Roy N \& Vetharaniam I (2011) Opportunities for predictive modelling and gut health. Conceptually exploring an in silico tool for quantifying the benefits of dietary fibre consumption. Agro Food Industry Hi-Tech 22: 18-22.

Rosendale DI, Blatchford PA, Sims IM, Parkar SG, Carnachan SM, Hedderley D \& Ansell J (2012) Characterizing kiwifruit carbohydrate utilization in vitro and its consequences for human faecal microbiota. Journal of Proteome Research 11: 5863-5875.

RStudio (2012) RStudio: Integrated development environment for R (Version 0.97.551) [Computer software]. Boston, MA. http://www.rstudio.org/.

Sauvageau J, Hinkley SF, Carnachan SM \& Sims IM (2010) Characterisation of polysaccharides from gold kiwifruit (Actinidia chinensis Planch. 'Hort16A'). Carbohydr Polym 82: 1110-1115.

Seager NG \& Haslemore RM (1993) Rapid estimation of fruit starch and soluble sugar concentrations in kiwifruit. Hortscience 28: 948-950.

Shahidi F (2009) Nutraceuticals and functional foods: whole versus processed foods. Trends in Food Science \& Technology 20: 376-387. 
542 Tannock GW, Munro K, Harmsen HJ, Welling GW, Smart J \& Gopal PK (2000) Analysis of the fecal

543 microflora of human subjects consuming a probiotic product containing Lactobacillus rhamnosus

544 DR20. Appl Environ Microbiol 66: 2578-2588.

545 Tuomilehto J, Lindstrom J, Eriksson JG, et al. (2001) Prevention of type 2 diabetes mellitus by

546 changes in lifestyle among subjects with impaired glucose tolerance. New England Journal of

547 Medicine 344: 1343-1350.

548 van der Sluis AA, Dekker M, Skrede G \& Jongen WMF (2002) Activity and concentration of

549 polyphenolic antioxidants in apple juice. 1. Effect of existing production methods. Journal of

550 Agricultural and Food Chemistry 50: 7211-7219.

551 Vazquez-Baeza Y, Pirrung M, Gonzalez A \& Knight R (2013) EMPeror: a tool for visualizing high-

552 throughput microbial community data. GigaScience 2: 16.

553 Wang Q, Garrity GM, Tiedje JM \& Cole JR (2007) Naive Bayesian classifier for rapid assignment of

554 rRNA sequences into the new bacterial taxonomy. Appl Environ Microbiol 73: 5261-5267.

555 Wolfe AJ (2005) The acetate switch. Microbiology and molecular biology reviews : MMBR 69: 12-50.

556 Wong JMW, de Souza R, Kendall CWC, Emam A \& Jenkins DJA (2006) Colonic health: Fermentation

557 and short chain fatty acids. Journal of Clinical Gastroenterology 40: 235-243.

558 Xu J, Mahowald MA, Ley RE, et al. (2007) Evolution of symbiotic bacteria in the distal human

559 intestine. Plos Biology 5: 1574-1586.

560

561

562

563

564

565

566

567

568

569

570

571

572

573 
Figures:
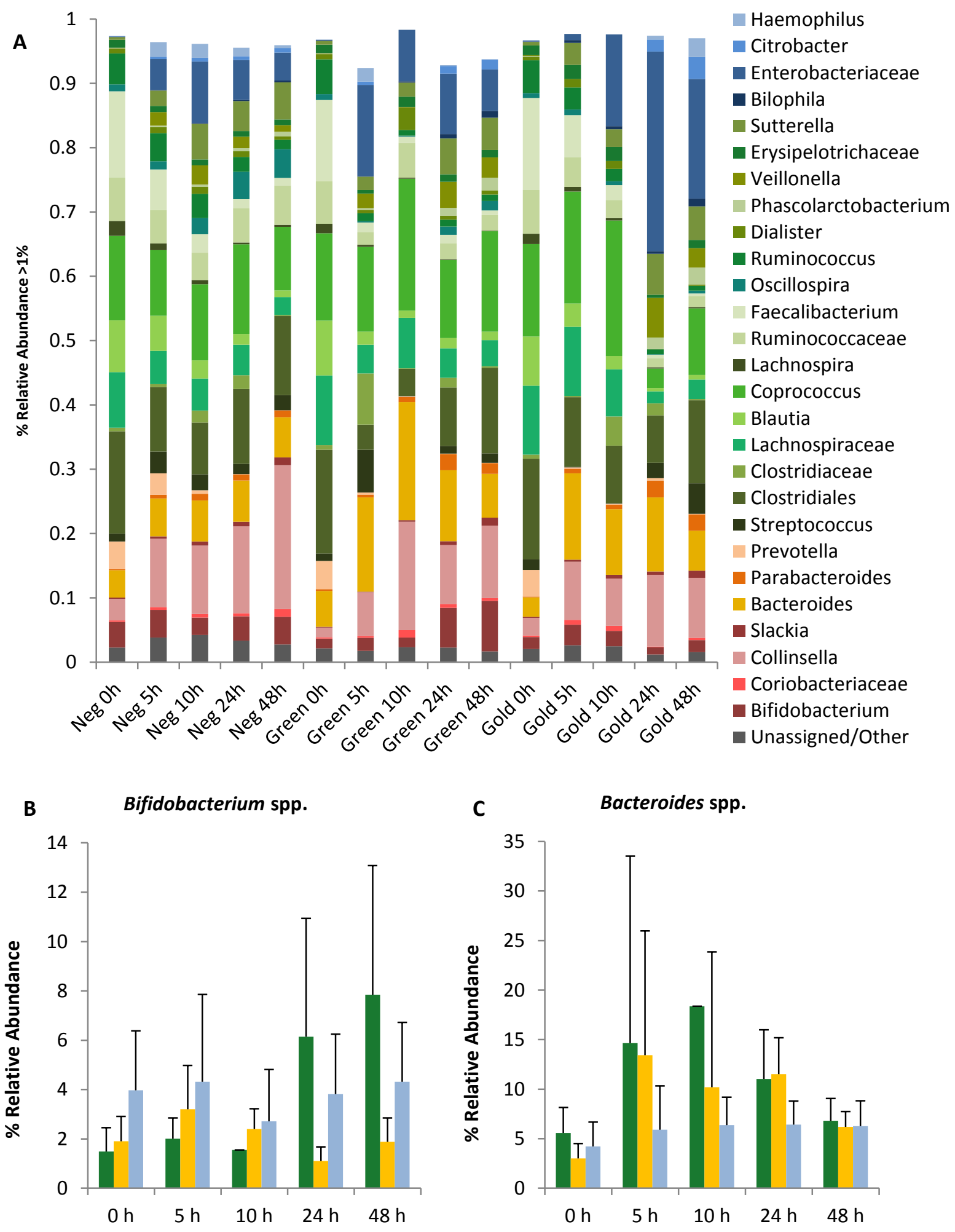
581 Figure 1 - Microbial composition of samples at all time points as determined by 16S rRNA gene sequencing. (A) Mean $(n=3)$. The four most abundant phyla are depicted as four colours Actinobacteria (red), Bacteroidetes (yellow), Firmicutes (green) and Proteobacteria (blue) with family and genus level taxonomy portrayed as differing shades of those colours within each phylum. Only genera of greater than $1 \%$ total read composition were included in this graph. (B) Relative abundance of Bifidobacterium spp. and (C) Bacteroides spp.. Green, gold and blue bars represent green kiwifruit, gold kiwifruit and the negative control respectively. Error bars are the SEM of the three fermentations.

588 SEM are displayed as error bars $(n=3)$.

589

590

591

592

593

594

595

596

597

598

599

600

601

602 


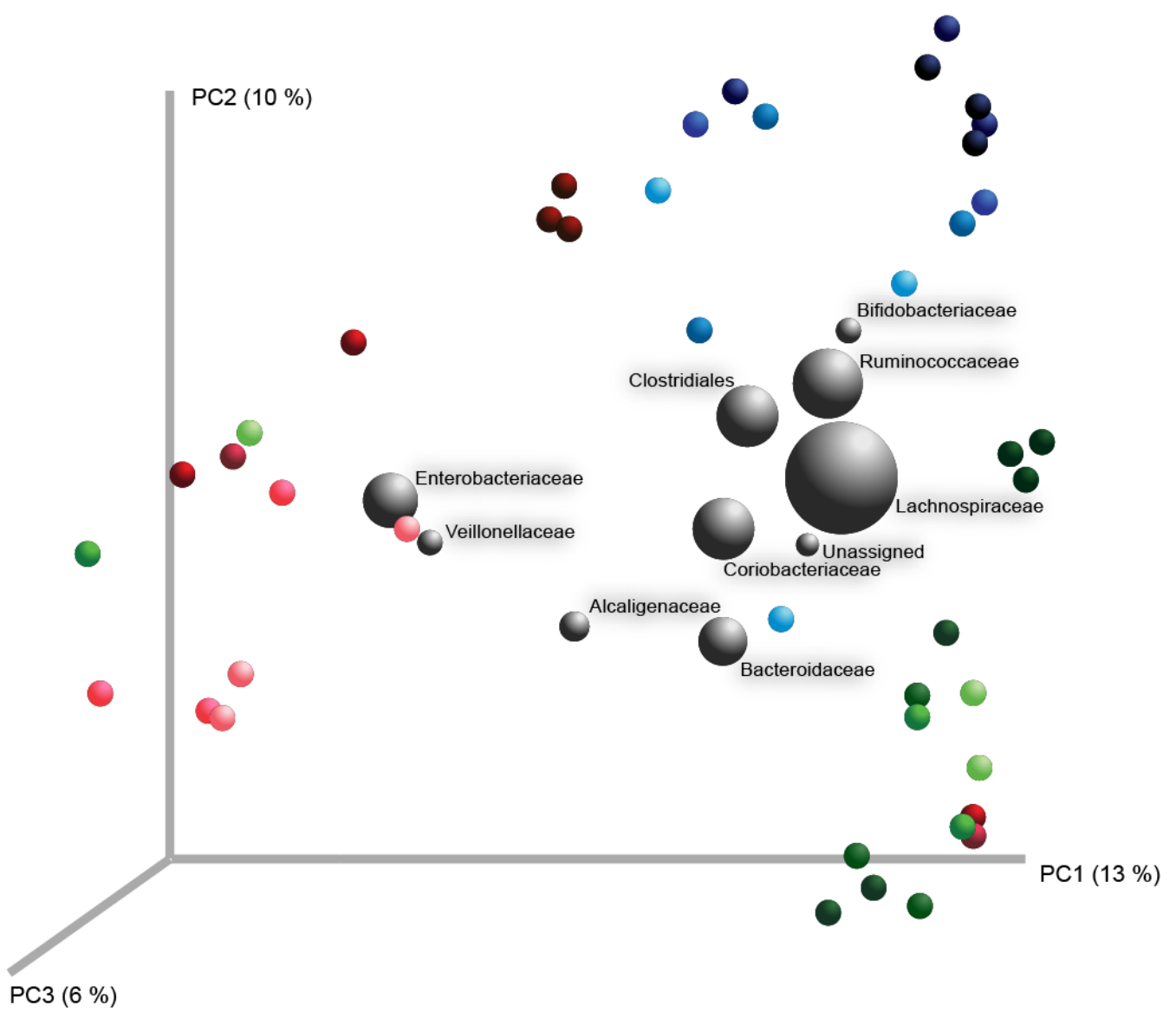


620 Figure 2 - Jackknifed beta diversity PCoA biplots showing unweighted UniFrac distances. 621 Plotted by faecal donor (fermentation), where donor 1,2 and 3 are shown with blue, green and red 622 spheres, respectively. Within the three colours, the fermentations are plotted by time, with darker 623 hues moving to lighter hues from $0 \mathrm{~h}, 5 \mathrm{~h}, 10 \mathrm{~h}, 24 \mathrm{~h}$, and $48 \mathrm{~h}$. Vector loadings (or weighting) by 624 bacteria at the family level are shown with grey spheres, the size of which corresponds to their 625 contribution to differentiation in the plot. 


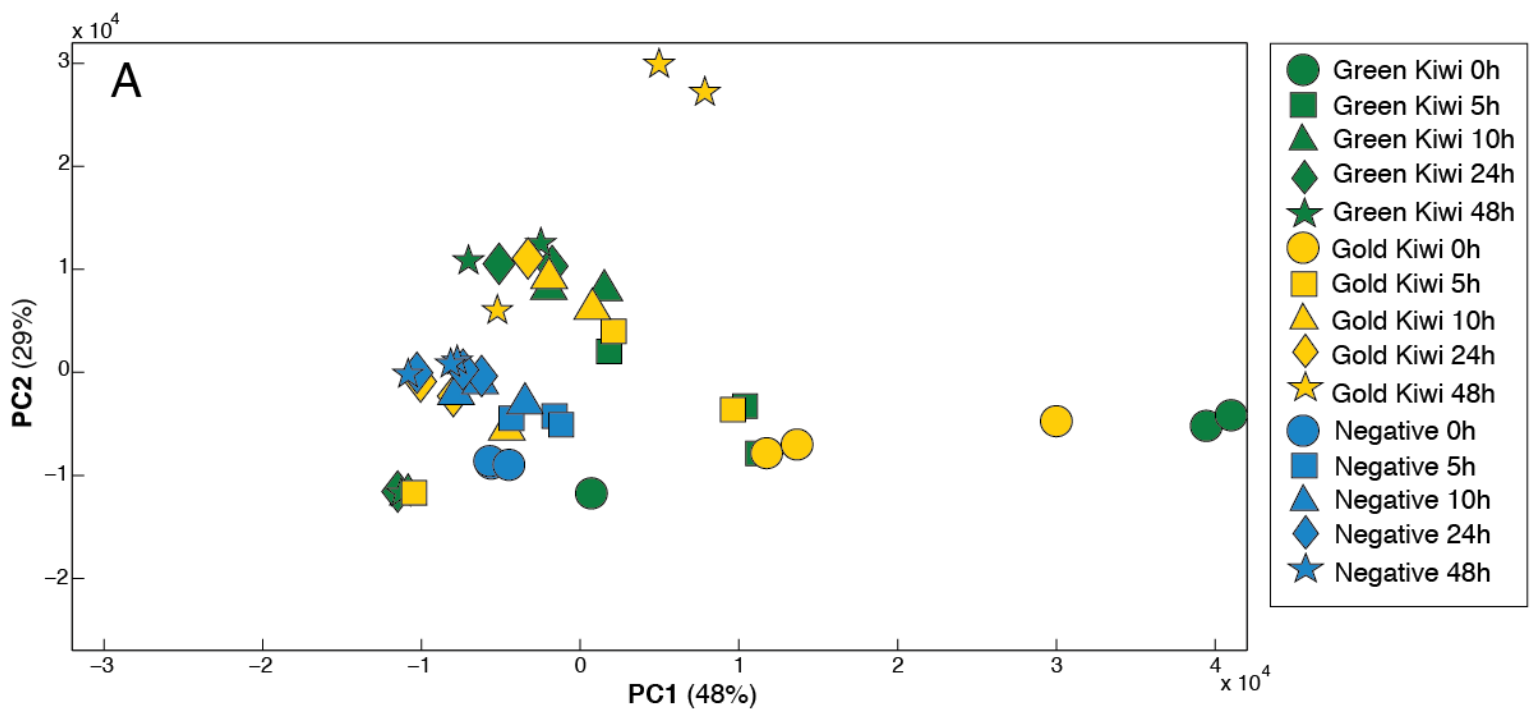

633

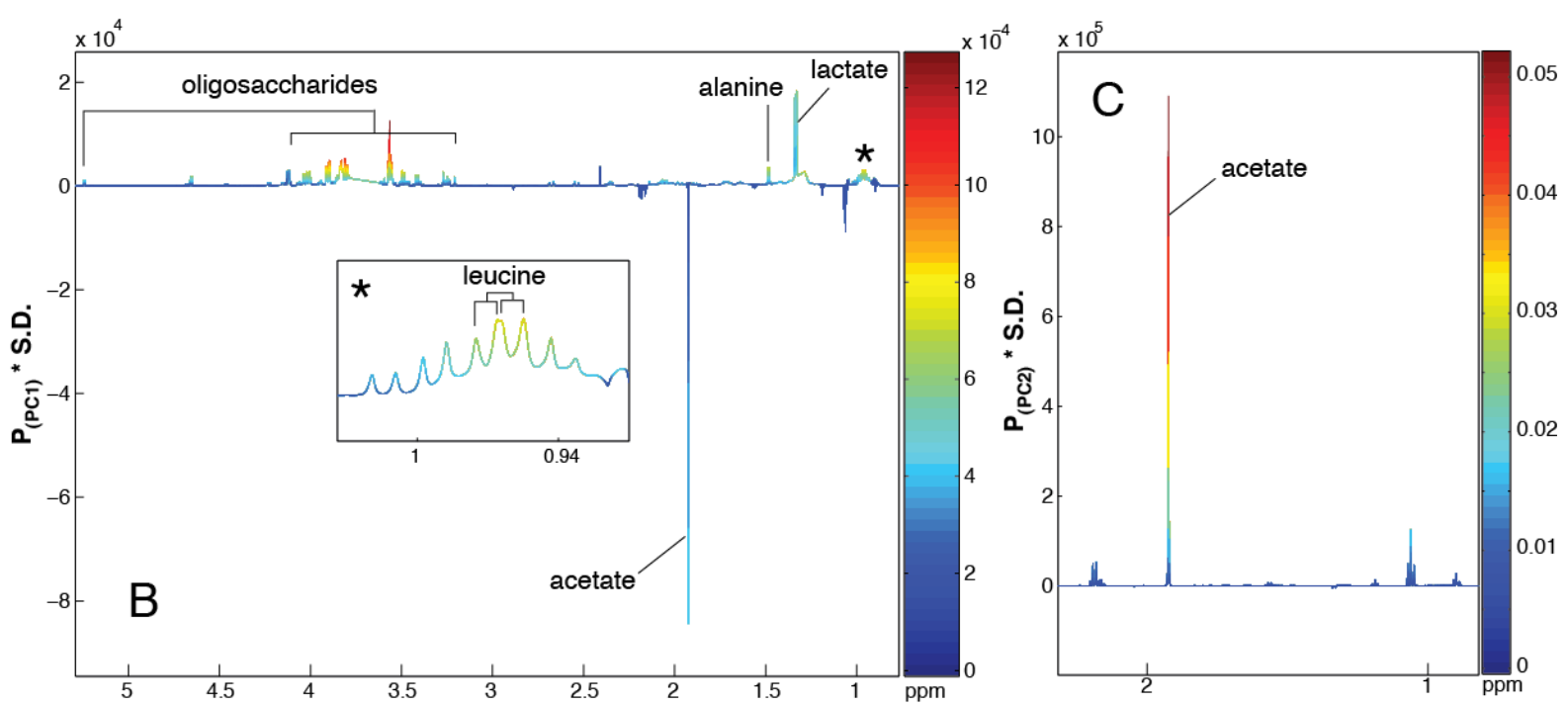


646

647

648

649

650

651

652

653

654

655

656

657

658

659

660

661

662

663

664

665

666

667

668

669

670

671
Figure 3: Principal Component Analysis (PCA) model of the metabolic profiles of all batch culture supernatants. (A) Scores plot for PC1 vs PC2 (\% variance explained in parenthesis). Product of PC loadings with standard deviation of the entire data set is plotted and coloured by the square of the PC for (B) PC1 and (C) PC2. 


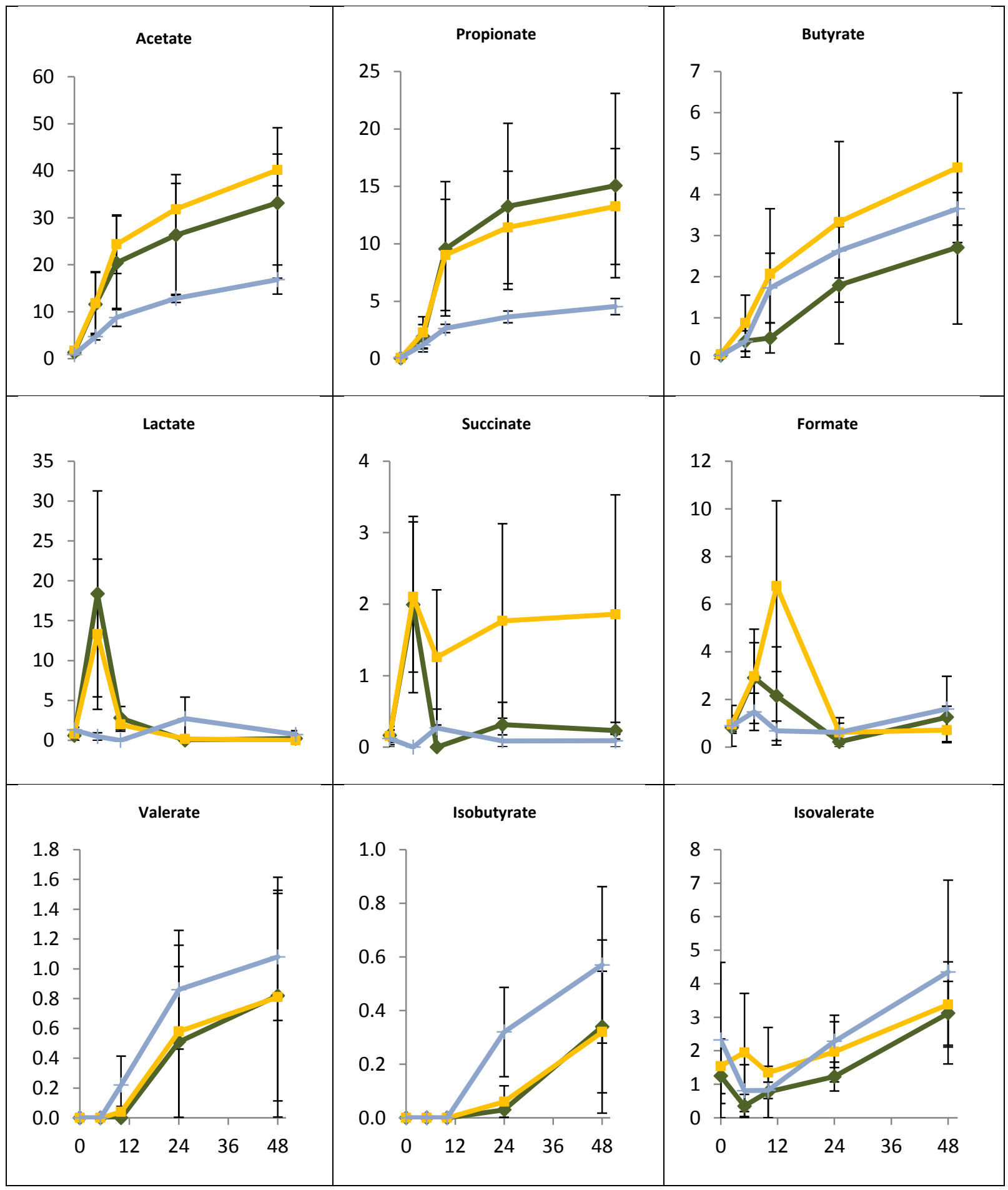

672

673

674

675

676 
677 Figure 4 - Short-chain fatty acid and organic acid production as determined by GC-FID

678 analysis. The units of the vertical axes are concentration ( $\mu \mathrm{mol} / \mathrm{mL}$ fermenta) and the horizontal axes

679 are time (hours). Green kiwifruit (green line), gold kiwifruit (gold line), negative control (blue line). SEM 680 are displayed as error bars $(n=3)$.

681 